AIP/123-QED

\title{
Classification of bi-qutrit positive partial transpose entangled edge states by their ranks
}

Seung-Hyeok Kye ${ }^{1}$ and Hiroyuki Osaka ${ }^{2}$

1) Department of Mathematics and Institute of Mathematics, Seoul National University, Seoul 151-742, Korea

${ }^{2)}$ Department of Mathematical Sciences, Ritsumeikan University, Kusatsu, Shiga, 525-8577 Japan

(Dated: 31 October 2018)

We construct $3 \otimes 3$ PPT entangled edge states with maximal ranks, to complete the classification of $3 \otimes 3$ PPT entangled edge states by their types. The ranks of the states and their partial transposes are 8 and 6 , respectively. These examples also disprove claims in the literature.

PACS numbers: 03.67.-a, 03.67.Hk, 03.65.Fd

Keywords: positive partial transposes, separable states, entanglement, edge states, product vectors 


\section{INTRODUCTION}

Let $M_{n}$ denote the $C^{*}$-algebra of all $n \times n$ matrices over the complex field, with the cone $M_{n}^{+}$of all positive semi-definite matrices. A positive semi-definite matrix in $M_{m} \otimes M_{n}$ is said to be separable if it is the convex sum of rank one projectors onto product vectors $x \otimes y \in \mathbb{C}^{m} \otimes \mathbb{C}^{n}$. A positive semi-definite matrix in $M_{m} \otimes M_{n}$ is said to be entangled if it is not separable. Since the convex cone of all separable ones coincides with $M_{m}^{+} \otimes M_{n}^{+}$, the entanglement consists of $\left(M_{m} \otimes M_{n}\right)^{+} \backslash M_{m}^{+} \otimes M_{n}^{+}$. The notion of entanglement is a unique phenomenon in non-commutative order structures, and there is no counterpart in classical mechanics. Indeed, it is well-known that the equality $(\mathcal{A} \otimes \mathcal{B})^{+}=\mathcal{A}^{+} \otimes \mathcal{B}^{+}$holds for commutative $C^{*}$-algebras $\mathcal{A}$ and $\mathcal{B}$ which are mathematical frameworks for classical mechanics. This notion of quantum entanglement has been one of the key research topics since the nineties, in relation with possible applications to quantum information theory and quantum computation theory.

One of the main research topics in the theory of entanglement is to distinguish entanglement from separability. If we take a rank one projector onto a product vector $x \otimes y$, then it is easy to see that its partial transpose is also a rank one projector onto the product vector $\bar{x} \otimes y$, where $\bar{x}$ denotes the vector whose entries are complex conjugates of the corresponding entries of the vector $x \in \mathbb{C}^{m}$. Recall that the partial transpose $(X \otimes Y)^{\tau}$ is given by $X^{\mathrm{t}} \otimes Y$ with the usual transpose $X^{\mathrm{t}}$ of $X$. Therefore, if $A \in M_{m} \otimes M_{n}$ is separable, then its partial transpose $A^{\tau}$ is also positive semi-definite, as was observed by Choi ${ }^{9}$ and Peres $\underline{25}$. A block matrix $A \in M_{m} \otimes M_{n}$ is said to be of PPT (positive partial transpose) if both of $A$ and $A^{\tau}$ are positive semi-definite. The notion of PPT turns out be to very important in quantum physics in relation with bound entanglement. See Ref. 16.

Woronowicz ${ }^{33}$ showed that if $m=2$ and $n \leq 3$ then the notions of separability and PPT coincide, and gave an explicit example of entanglement $A \in M_{2} \otimes M_{4}$ which is of PPT. This kind of block matrix is called a PPTES (positive partial transpose entangled state) when it is normalized. The first example of PPTES in $M_{3} \otimes M_{3}$ was found by Choi ${ }^{9}$. A PPTES $A$ is said to be a PPT entangled edge state, or just an edge state in short, if there exists no nonzero product vector $x \otimes y \in \mathcal{R} A$ with $\bar{x} \otimes y \in \mathcal{R} A^{\tau}$ as was introduced in Ref. 23, where $\mathcal{R} A$ denotes the range space of $A$. In other words, edge states violate the range criterion for separability $\underline{18}$ in an extreme way. 
Since every PPT state is the convex sum of a separable state and an edge state, it is essential to classify edge states to understand the whole structures of PPT states. The first step to classify them is to use the ranks. A PPT state $A$ is said to be of type $(p, q)$ if the rank of $A$ is $p$ and the rank of $A^{\tau}$ is $q$, as was introduced in Ref. 26. Now, we concentrate on the case of $3 \otimes 3$. By the results in Refs. 5, 19, 20, and 28, we have the following possibilities of types for $3 \otimes 3$ PPT entangled edge states:

$$
(4,4),(5,5),(6,5),(7,5),(8,5),(6,6),(7,6),(8,6) \text {, }
$$

here we list up types $(p, q)$ with $p \geq q$ by the symmetry. See Ref. 3, 9-11, 13, 14, 18, and 31 for concrete examples of $3 \otimes 3$ edge states of various types. We refer to Ref. 20 for a summary of examples. All possibilities have been realized in the literature mentioned above, except for the case of $(8,6)$. In fact, it has been claimed in Ref. 26 that if there is a $3 \otimes 3$ PPT entangled edge state of type $(p, q)$ then $p+q \leq 13$.

The purpose of this note is to present two parameterized examples of $3 \otimes 3$ PPT entangled edge states of type $(8,6)$, to complete the classification of $3 \otimes 3$ edges states by their types. These examples disprove the above mentioned claim ${ }^{26}$. Our examples also disprove another $\operatorname{claim}^{22}$ that if $D=(\mathcal{R} A)^{\perp}$ and $E=\left(\mathcal{R} A^{\tau}\right)^{\perp}$ for a PPT state $A \in M_{m} \otimes M_{n}$ and $\operatorname{dim} D+$ $\operatorname{dim} E=m+n-2$, then there exist finitely many product vectors $x \otimes y \in \mathcal{R} A$ with $\bar{x} \otimes y \in \mathcal{R} A^{\tau}$.

After we explain in the next section the notion of PPT edge states in the context of the whole convex structures of the convex cone generated by PPT states, we present our construction of two parameterized examples of edges of type $(8,6)$ in the Section 3 . In the last section, we also exhibit various types of edge states arising from this construction.

\section{CONVEX GEOMETRY OF PPT STATES}

We denote by $\mathbb{V}_{1}$ and $\mathbb{T}$ the convex cones generated by all separable and PPT states, respectively. The PPT criterion by Choi and Peres tells us that the relation $\mathbb{V}_{1} \subset \mathbb{T}$ holds. One of the best way to understand the whole structures of a given convex set is to characterize the lattice of all faces. We have very few general information for the facial structures of the convex cone $\mathbb{V}_{1}$ itself. See Ref. 1 in this direction. On the other hand, we have an easy way to describe faces of the cone $\mathbb{T}$ generated by PPT states. 
Every faces of the cone $\mathbb{T}$ is determined 12 by a pair of subspaces of $\mathbb{C}^{m} \otimes \mathbb{C}^{n}$. More precisely, every face of $\mathbb{T}$ is of the form

$$
\sigma(D, E)=\left\{A \in \mathbb{T}: \mathcal{R} A \subseteq D, \mathcal{R} A^{\tau} \subseteq E\right\}
$$

for a pair $(D, E)$ of subspaces of $\mathbb{C}^{m} \otimes \mathbb{C}^{n}$. Nevertheless, it is very difficult in general to determine which pairs of subspaces give rise to faces of the convex cone $\mathbb{T}$, and this difficulty is one of the main motivation of this note. In the case of $2 \otimes 2$, all faces of $\mathbb{T}$ have been found 12 in terms of pairs of subspaces, using the facial structures $\frac{4}{30}$ of the convex cone of all positive linear maps between $M_{2}$. Recall that a point $x$ of a convex set $C$ is said to be an interior point of $C$ if the line segment from any point of $C$ to $x$ may be extended within $C$. The set of all interior point of $C$ will be denoted by $\operatorname{int} C$, which is nothing but the relative interior of $C$ with respect to the affine manifold generated by $C$. Note that int $C$ is never empty for any convex set $C$. A point of $C$ which is not an interior point is said to be a boundary point. The set of all boundary points of $C$ will be denoted by $\partial C$. We recall that the interior of $\sigma(D, E)$ is given by

$$
\operatorname{int} \sigma(D, E)=\left\{A \in \mathbb{T}: \mathcal{R} A=D, \mathcal{R} A^{\tau}=E\right\}
$$

From now on, we compare boundary structures of the two convex cones $\mathbb{V}_{1}$ and $\mathbb{T}$. Basically, we have the following four cases for a given face $\sigma(D, E)$ of the cone $\mathbb{T}$ :

- $\sigma(D, E) \subseteq \mathbb{V}_{1}$.

- $\sigma(D, E) \nsubseteq \mathbb{V}_{1}$ but int $\sigma(D, E) \cap \mathbb{V}_{1} \neq \emptyset$.

- $\operatorname{int} \sigma(D, E) \cap \mathbb{V}_{1}=\emptyset$ but $\partial \sigma(D, E) \cap \mathbb{V}_{1} \neq \emptyset$.

- $\sigma(D, E) \cap \mathbb{V}_{1}=\emptyset$.

Recall that the range criterion for separability tells us that if a PPT state $A$ is separable with $D=\mathcal{R} A$ and $E=\mathcal{R} A^{\tau}$ then there exist product vectors $x_{\iota} \otimes y_{\iota} \in \mathbb{C}^{m} \otimes \mathbb{C}^{n}$ such that

$$
D=\operatorname{span}\left\{x_{\iota} \otimes y_{\iota}\right\}, \quad E=\operatorname{span}\left\{\bar{x}_{\iota} \otimes y_{\iota}\right\}
$$

We say that a pair $(D, E)$ satisfies the range criterion if there exist product vectors with the above property. Therefore, we see that if the interior of $\sigma(D, E)$ has a nonempty intersection with the cone $\mathbb{V}_{1}$ then $(D, E)$ satisfies the range criterion. The converse of this statement is 
also true as was shown in Ref. 7, even though the converse of the range criterion itself does not hold. In short, we see that $(D, E)$ satisfies the range criterion if and only if the first two conditions among the above four hold. In terms of a PPT state $A$ itself, we see that $\left(\mathcal{R} A, \mathcal{R} A^{\tau}\right)$ satisfies the range criterion if and only if the smallest face containing $A$ has a separable state in its interior. Recall that every point $x$ of a convex set determines a unique face in which $x$ is an interior point. This is the smallest face containing $x$.

It remains two cases to be considered: A face $\sigma(D, E)$ either touches the cone $\mathbb{V}_{1}$ at the boundary or never touches the cone $\mathbb{V}_{1}$. It is easy to see that the latter case occurs if and only if every element of the face $\sigma(D, E)$ is a PPT entangled edge state. If this is the case with $\operatorname{dim} D=p$ and $\operatorname{dim} E=q$ then every interior point of the face $\sigma(D, E)$ is an edge state of type $(p, q)$, and every boundary point of $\sigma(D, E)$ is also an edge state of type $(s, t)$ with $s<p$ or $t<q$.

The first step to characterize the lattice of all faces of the cone $\mathbb{T}$ is to find all pairs $(p, q)$ of natural numbers for which there exists a face $\sigma(D, E)$ with $\operatorname{dim} D=p$ and $\operatorname{dim} E=q$. See Ref. 22 for this line of research. This classification is especially important for the cases of separable states and edge states, since every PPT state is the sum of a separable state and an edge state. This task for separable states is nothing but to classify the dimensions of pairs of subspaces satisfying the range criterion. In the case $2 \otimes n$, all pairs $(p, q)$ of natural numbers have been characterized ${ }^{7}$ for which there exist pairs $(D, E)$ satisfying the range criterion with $\operatorname{dim} D=p$ and $\operatorname{dim} E=q$.

As for edge states, there are previous results in the literature in two directions. It was shown 19,24 that if $A$ is supported on $\mathbb{C}^{m} \otimes \mathbb{C}^{n}$ and the rank of $A \in M_{m} \otimes M_{n}$ is less than or equal to $\max \{m, n\}$, then two notions of PPT and separability coincide. This gives a lower bound for the ranks of $A$ and $A^{\tau}$ for an edge state $A \in M_{m} \otimes M_{n}$ : If $A$ is an $m \otimes n$ edge of type $(p, q)$, then we have

$$
p, q>\max \{m, n\}
$$

On the other hand, for a given pair $(D, E)$ of subspaces in $\mathbb{C}^{m} \otimes \mathbb{C}^{n}$, it was shown ${ }^{20}$ that there must exist $x \otimes y \in D$ with $\bar{x} \otimes y \in E$, whenever either the inequality

$$
\operatorname{dim} D+\operatorname{dim} E>2 m n-m-n+2
$$


holds, or $\operatorname{dim} D+\operatorname{dim} E=2 m n-m-n+2$ and

$$
\sum_{r+s=m-1}(-1)^{r}\left(\begin{array}{l}
k \\
r
\end{array}\right)\left(\begin{array}{l}
\ell \\
s
\end{array}\right) \neq 0
$$

hold with $k=\operatorname{dim} D^{\perp}$ and $\ell=\operatorname{dim} E^{\perp}$. This gives us an upper bound for the ranks of $A$ and $A^{\tau}$ for an edge state $A \in M_{m} \otimes M_{n}$.

In case of $m=n=3$, we have $2 m n-m-n+2=14$. It is easy to see that $(k, \ell)=(2,2)$ satisfies the above condition, but $(k, \ell)=(1,3)$ does not satisfy. Furthermore, it is now known 5,28 that every PPT entanglement of rank 4 is automatically of type $(4,4)$. All of these arguments give us the possibilities of types as is given in (11). See also Ref. 20 for the summary in the case of $(m, n)=(2,4)$ as well as in the case of $m=n=3$. It is unknown whether there exists a $2 \otimes 4$ PPT edge state of type $(6,6)$ or not. Classifications of possible types of edge states for the $2 \otimes 4$ and $3 \otimes 3$ cases are summarized in the following pictures:
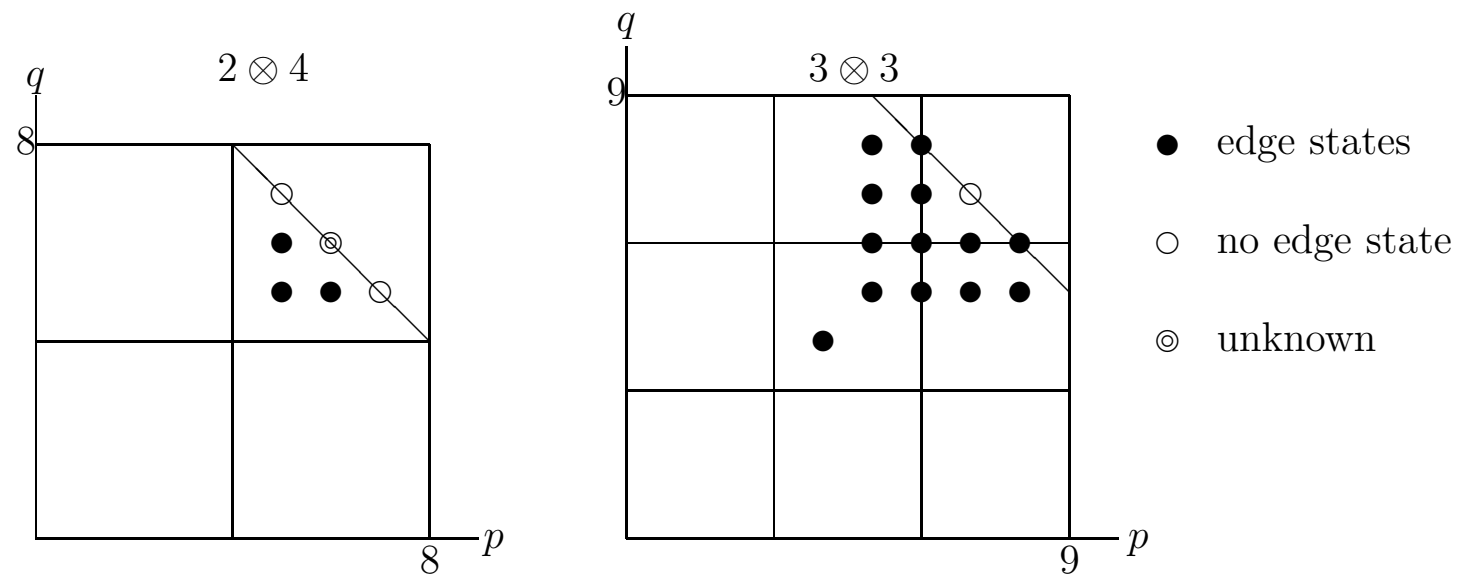

\section{CONSTRUCTION}

We begin with the following $3 \times 3$ matrix

$$
P[\theta]:=\left(\begin{array}{ccc}
e^{i \theta}+e^{-i \theta} & -e^{i \theta} & -e^{-i \theta} \\
-e^{-i \theta} & e^{i \theta}+e^{-i \theta} & -e^{i \theta} \\
-e^{i \theta} & -e^{-i \theta} & e^{i \theta}+e^{-i \theta}
\end{array}\right)
$$

which has a kernel vector $(1,1,1)^{\mathrm{t}}$. Considering the principal submatrices, we see that $P[\theta]$ is positive semi-definite if and only if $\cos \theta \geq 0$ and $2 \cos 2 \theta \geq-1$ if and only if $-\frac{\pi}{3} \leq \theta \leq \frac{\pi}{3}$. If $-\frac{\pi}{3}<\theta<\frac{\pi}{3}$ then $P[\theta]$ is of rank two, and if $\theta=-\frac{\pi}{3}$ or $\theta=\frac{\pi}{3}$ then $P[\theta]$ is of rank one. 
Consider the following matrix

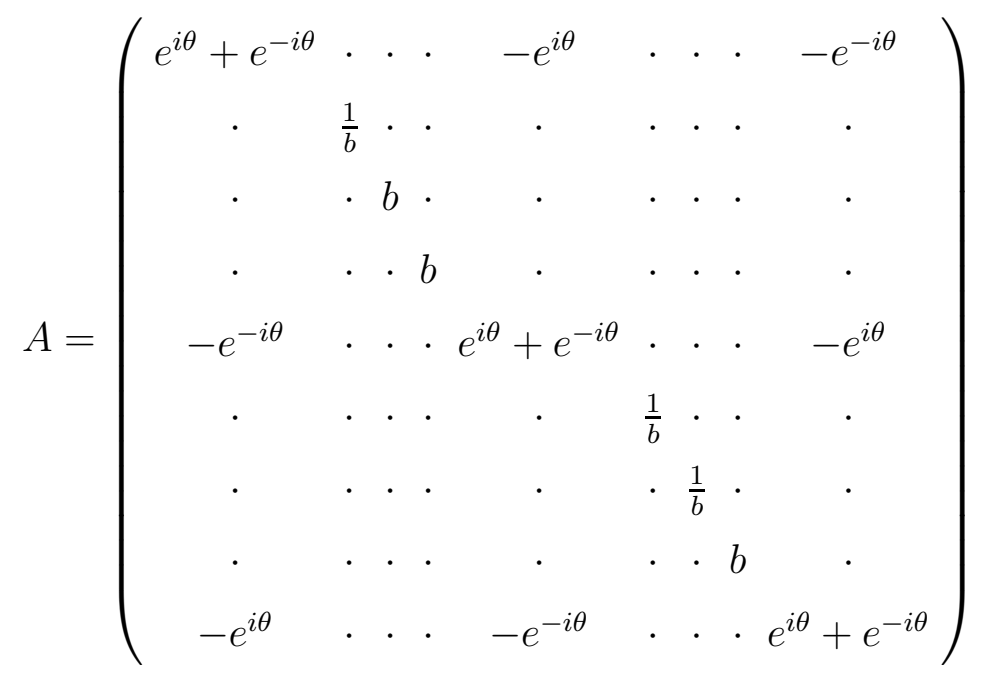

in $M_{3} \otimes M_{3}$ with the conditions

$$
b>0, \quad-\frac{\pi}{3}<\theta<\frac{\pi}{3}, \quad \theta \neq 0,
$$

where denote zero. The partial transpose $A^{\tau}$ of $A$ is given by

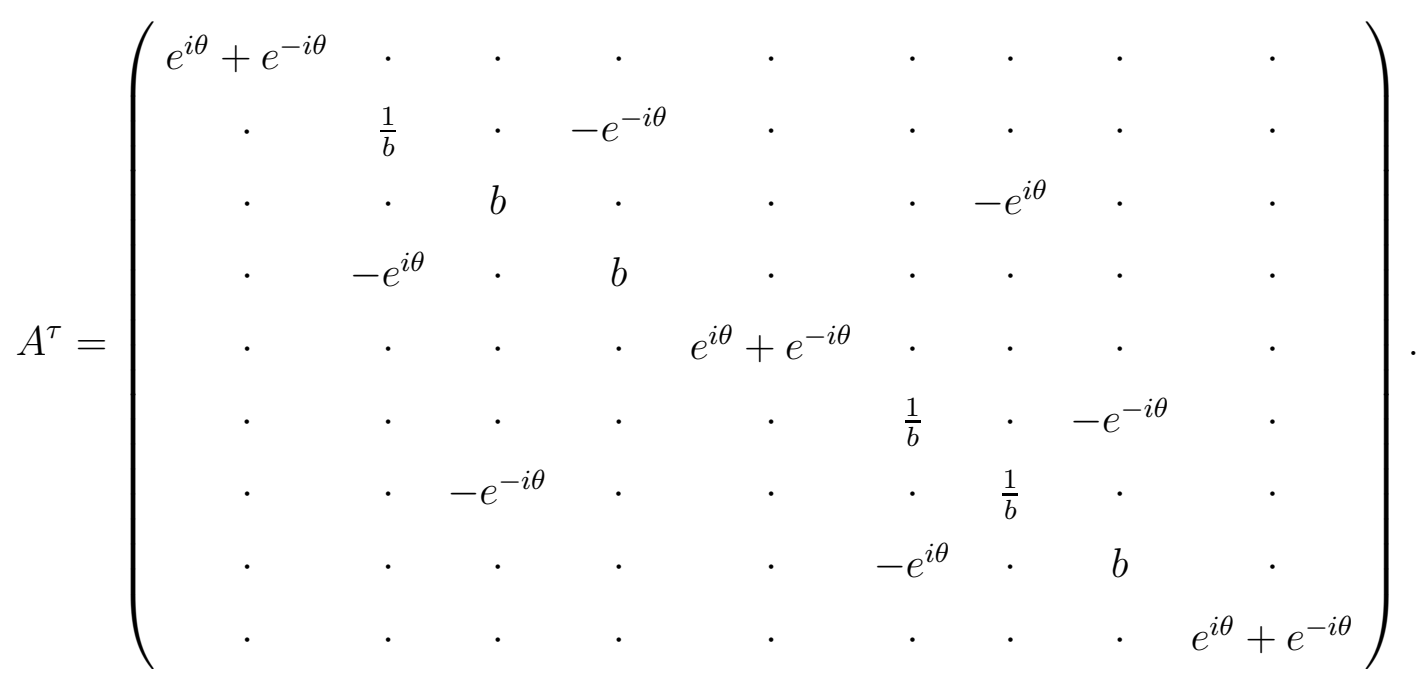

It is clear that $A$ is of PPT under the condition (3), and we have $\operatorname{rank} A=8$ and $\operatorname{rank} A^{\tau}=6$.

We proceed to show that $A$ is a PPT entangled edge state under the condition (3). First of all, we note that the kernel of $A$ is spanned by

$$
(1,0,0 ; 0,1,0 ; 0,0,1)^{\mathrm{t}}
$$

and the kernel of $A^{\tau}$ is spanned by the following three vectors:

$$
\begin{aligned}
& \left(0, b, 0 ; e^{i \theta}, 0,0 ; 0,0,0\right)^{\mathrm{t}}, \\
& \left(0,0,0 ; 0,0, b ; 0, e^{i \theta}, 0\right)^{\mathrm{t}}, \\
& \left(0,0, e^{i \theta} ; 0,0,0 ; b, 0,0\right)^{\mathrm{t}} .
\end{aligned}
$$


Suppose that a product vector $z=x \otimes y \in \mathbb{C}^{3} \otimes \mathbb{C}^{3}$ is in the range of $A$, and $\bar{x} \otimes y$ is in the range of $A^{\tau}$. Then we have

$$
x_{1} y_{1}+x_{2} y_{2}+x_{3} y_{3}=0
$$

and

$$
\begin{aligned}
& b \bar{x}_{1} y_{2}+e^{-i \theta} \bar{x}_{2} y_{1}=0, \\
& b \bar{x}_{2} y_{3}+e^{-i \theta} \bar{x}_{3} y_{2}=0 \\
& b \bar{x}_{3} y_{1}+e^{-i \theta} \bar{x}_{1} y_{3}=0 .
\end{aligned}
$$

From (5) we see that at least one of $x_{i}, y_{i}$ is zero. Indeed, we have

$$
b^{3} \bar{x}_{1} \bar{x}_{2} \bar{x}_{3} y_{1} y_{2} y_{3}=-e^{-3 i \theta} \bar{x}_{1} \bar{x}_{2} \bar{x}_{3} y_{1} y_{2} y_{3}
$$

by (5), from which $\bar{x}_{1} \bar{x}_{2} \bar{x}_{3} y_{1} y_{2} y_{3}=0$. If $x \otimes y$ is nonzero, then we also have $x_{i}=0 \Longleftrightarrow y_{i}=0$ from (15).

We first consider the case of $x_{3}=y_{3}=0$. Then we have

$$
x_{1} y_{1}+x_{2} y_{2}=0, \quad b \bar{x}_{1} y_{2}+e^{-i \theta} \bar{x}_{2} y_{1}=0,
$$

from which we have

$$
\bar{x}_{1} x_{1} y_{1}+\bar{x}_{1} x_{2} y_{2}=0, \quad b \bar{x}_{1} x_{2} y_{2}+e^{-i \theta} \bar{x}_{2} x_{2} y_{1}=0 .
$$

Therefore, we get

$$
\left|x_{1}\right|^{2} y_{1}=-\bar{x}_{1} x_{2} y_{2}=\frac{e^{-i \theta}}{b}\left|x_{2}\right|^{2} y_{1} .
$$

Since $\theta \neq 0$, we conclude that $x_{1}=x_{2}=0$ or $y_{1}=0$. If $x_{1}=x_{2}=0$, then $x=0$. If $y_{1}=0$ and either $x_{1}$ or $x_{2}$ is nonzero, then we have $y=0$. Similar arguments for the cases $x_{1}=y_{1}=0$ and $x_{2}=y_{2}=0$ show that if $x, y \in \mathbb{C}^{3}$ satisfy the relations (41) and (5)), then $x \otimes y=0$. This shows that there exists no nonzero product vector $x \otimes y \in \mathcal{R} A$ with $\bar{x} \otimes y \in \mathcal{R} A^{\tau}$. Therefore, we conclude that $A$ is a PPT entangled edge state of type $(8,6)$.

Recall ${ }^{32}$ that every 5 -dimensional subspace of $\mathbb{C}^{3} \otimes \mathbb{C}^{3}$ has a product vector. This is equivalent to say that every system of equations consisting of four homogeneous linear equations with respect to unknowns $\left\{x_{i} y_{j}: i, j=1,2,3\right\}$ must have nontrivial solutions. But, the system of four equations from (44) and (51) involve complex conjugates, and may not have nonzero solutions. This seems to be the main point for the wrong statements in Ref. 22 and 26. 
For nonnegative real numbers $a, b$ and $c$, we consider the following linear map

$$
\Phi[a, b, c](X)=\left(\begin{array}{ccc}
a x_{11}+b x_{22}+c x_{33} & -x_{12} & -x_{13} \\
-x_{21} & c x_{11}+a x_{22}+b x_{33} & -x_{23} \\
-x_{31} & -x_{32} & b x_{11}+c x_{22}+a x_{33}
\end{array}\right)
$$

between $M_{3}$, as was introduced in Ref. 6. We also recall that the Choi matrix $C_{\phi} \in M_{m} \otimes M_{n}$ of a linear map $\phi: M_{m} \rightarrow M_{n}$ is given by

$$
C_{\phi}:=\sum_{i, j=1}^{m} e_{i j} \otimes \phi\left(e_{i j}\right) \in M_{m} \otimes M_{n},
$$

and $C_{\phi}$ is of PPT if and only if $\phi$ is both completely positive and completely copositive by Ref. 8. We also note that $\Phi[a, b, c]$ is both completely positive and completely copositive if and only if $a \geq 2$ and $b c \geq 1$ by Ref. 6. If $\theta=0$ then the matrix $A$ in (2) is just the Choi matrix of the map $\Phi\left[2, b, \frac{1}{b}\right]$, which is a PPT state of type $(8,6)$. On the other hand, we have the following PPT states

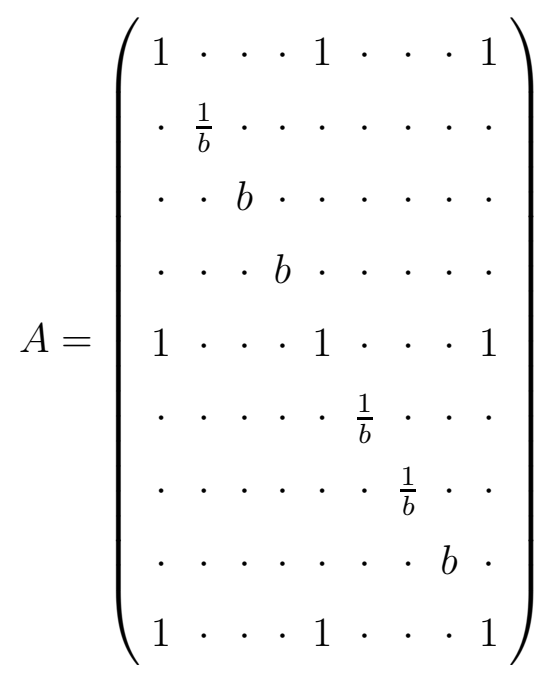

of type $(7,6)$ in the literature ${ }^{13}$, which is an edge state whenever $b>0$ and $b \neq 1$. The key idea of the construction was to parameterized offdiagonals -1 and 1 of these two cases by $e^{i \theta}$. We note that a variant of (6) has been used by Størmer ${ }^{31}$ to give a short proof of the indecomposability of the Choi map $\Phi[1,0, \lambda]$ for $\lambda \geq 1$.

If $\theta=0$ then $A$ in (2) turns out to be separable. Indeed, if we take product vectors

$$
\begin{aligned}
& z_{1}(\omega)=(0,1, \sqrt{b} \omega) \otimes(0, \sqrt{b},-\bar{\omega})=(0,0,0 ; 0, \sqrt{b},-\bar{\omega} ; 0, b \omega,-\sqrt{b}) \\
& z_{2}(\omega)=(\sqrt{b} \omega, 0,1) \otimes(-\bar{\omega}, 0, \sqrt{b})=(-\sqrt{b}, 0, b \omega ; 0,0,0 ;-\bar{\omega}, 0, \sqrt{b}) \\
& z_{3}(\omega)=(1, \sqrt{b} \omega, 0) \otimes(\sqrt{b},-\bar{\omega}, 0)=(\sqrt{b},-\bar{\omega}, 0 ; b \omega,-\sqrt{b}, 0 ; 0,0,0)
\end{aligned}
$$


in $\mathbb{C}^{3} \otimes \mathbb{C}^{3}$ then it is straightforward to see that

$$
A=\frac{1}{3 b} \sum_{i=1}^{3} \sum_{\omega \in \Omega} z_{i}(\omega) z_{i}(\omega)^{*}
$$

where $\Omega=\left\{1, e^{\frac{2}{3} \pi i}, e^{-\frac{2}{3} \pi i}\right\}$ is the third roots of unity. We note that the Choi matrix of the map $\Phi[a, b, c]$ is of PPT if and only if $a \geq 2$ and $b c \geq 1$, and so it is the sum of a diagonal matrix with nonnegative diagonal entries and a separable one. Therefore, we see that the Choi matrix of the map $\Phi[a, b, c]$ is of PPT if and only if it is separable. This shows that the linear map $\Phi[a, b, c]$ is super-positive in the sense of Ref. 2, or equivalently an entanglement breaking channel in the sense of Ref. 17 and 21 if and only if it is both completely positive and completely copositive if and only if $a \geq 2$ and $b c \geq 1$. See Ref. 27 for related topics.

If we put the following number

$$
a_{\theta}=\max \left\{e^{i\left(\theta+\frac{3}{2} \pi\right)}+e^{-i\left(\theta+\frac{3}{2} \pi\right)}, e^{i \theta}+e^{-i \theta}, e^{i\left(\theta-\frac{3}{2} \pi\right)}+e^{-i\left(\theta-\frac{3}{2} \pi\right)}\right\}
$$

in the place of $e^{i \theta}+e^{-i \theta}$ when we define the matrix $A$ in (2), then we have similar PPT edge states for every $\theta$. Note that $a_{\theta}$ is the smallest number so that

$$
\left(\begin{array}{ccc}
a_{\theta} & -e^{i \theta} & -e^{-i \theta} \\
-e^{-i \theta} & a_{\theta} & -e^{i \theta} \\
-e^{i \theta} & -e^{-i \theta} & a_{\theta}
\end{array}\right)
$$

is positive semi-definite.

\section{EDGE STATES OF OTHER TYPES}

Let $A$ be the matrix given by (21). Now, we search edge states $X$ in the smallest face containing $A$ by a similar method as in Ref. 13. Note that $X$ is in this face if and only if the relations

$$
\mathcal{R} X \subseteq \mathcal{R} A, \quad \mathcal{R} X^{\tau} \subseteq \mathcal{R} A^{\tau}
$$

hold. Note that every range vector of $A^{\tau}$ is of the form

$$
\left(\xi_{i}, \frac{\alpha_{i}}{\sqrt{b}},-\gamma_{i} \sqrt{b} e^{i \theta} ;-\alpha_{i} \sqrt{b} e^{i \theta}, \eta_{i}, \frac{\beta_{i}}{\sqrt{b}} ; \frac{\gamma_{i}}{\sqrt{b}},-\beta_{i} \sqrt{b} e^{i \theta}, \zeta_{i}\right)^{\mathrm{t}},
$$

for scalars $\xi_{i}, \eta_{i}, \zeta_{i}, \alpha_{i}, \beta_{i}$ and $\gamma_{i}$. We denote by $P$ the rank one projector onto the vector

$$
\left(1, \frac{1}{\sqrt{b}},-\sqrt{b} e^{i \theta} ;-\sqrt{b} e^{i \theta}, 1, \frac{1}{\sqrt{b}} ; \frac{1}{\sqrt{b}},-\sqrt{b} e^{i \theta}, 1\right)^{\mathrm{t}}
$$


and by $Q_{i}$ onto the vector

$$
\left(\xi_{i}, \alpha_{i}, \gamma_{i} ; \alpha_{i}, \eta_{i}, \beta_{i} ; \gamma_{i}, \beta_{i}, \zeta_{i}\right)^{\mathrm{t}}
$$

respectively. Here, the projector onto a column vector $w$ means the rank one matrix $w w^{*}$. Then we see that $A^{\tau}$ is the Hadamard product of $P$ and $\sum_{i} Q_{i}$ for suitable choice of $\xi_{i}, \eta_{i}, \zeta_{i}, \alpha_{i}, \beta_{i}$ and $\gamma_{i}$. If we write $\xi, \eta, \zeta, \alpha, \beta$ and $\gamma$ the vectors whose $i$-th entries are $\xi_{i}, \eta_{i}, \zeta_{i}, \alpha_{i}, \beta_{i}$ and $\gamma_{i}$, respectively, then the matrix $X=\left(X^{\tau}\right)^{\tau}$ is the Hadamard product of the following two matrices:

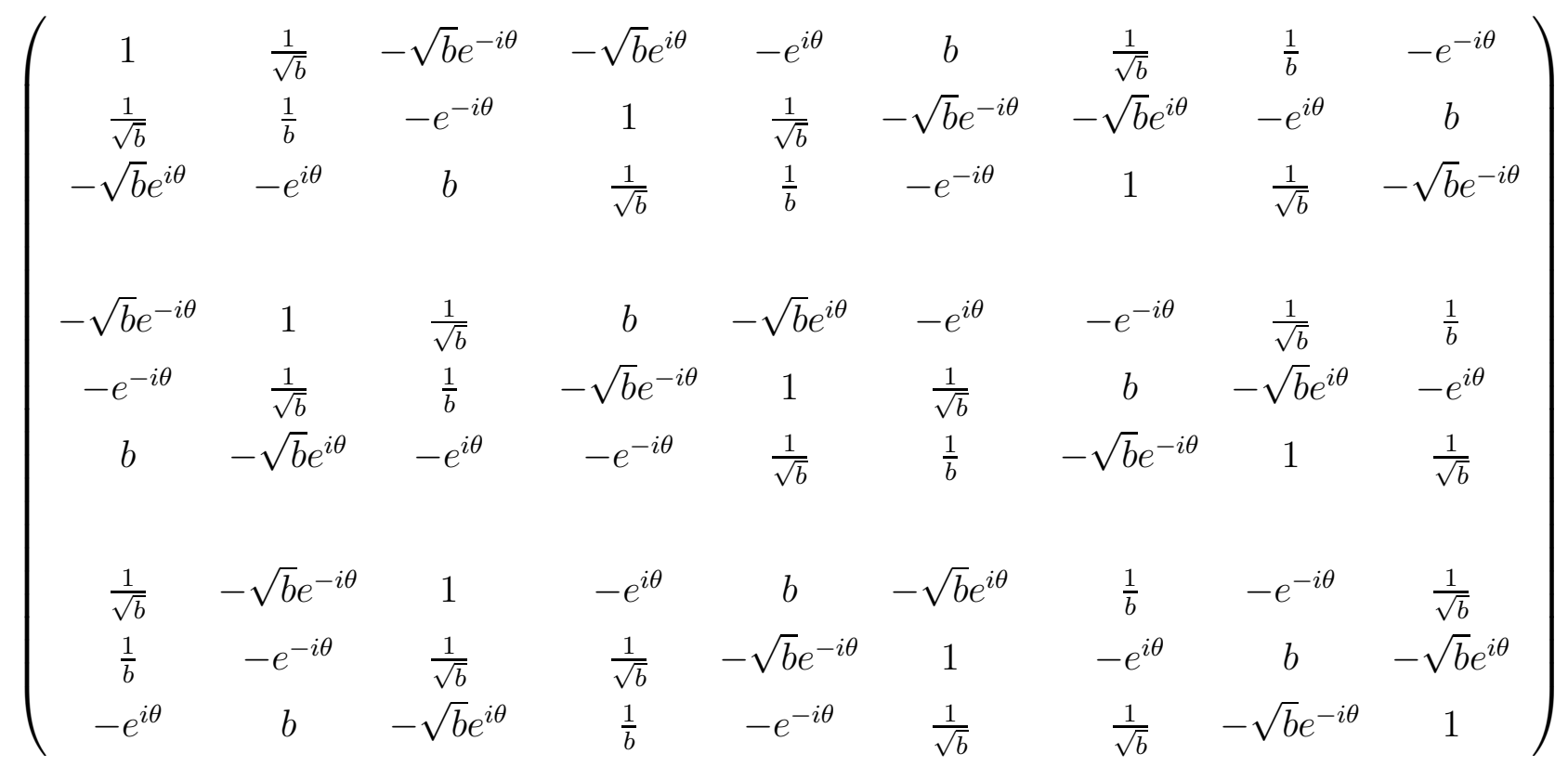

and

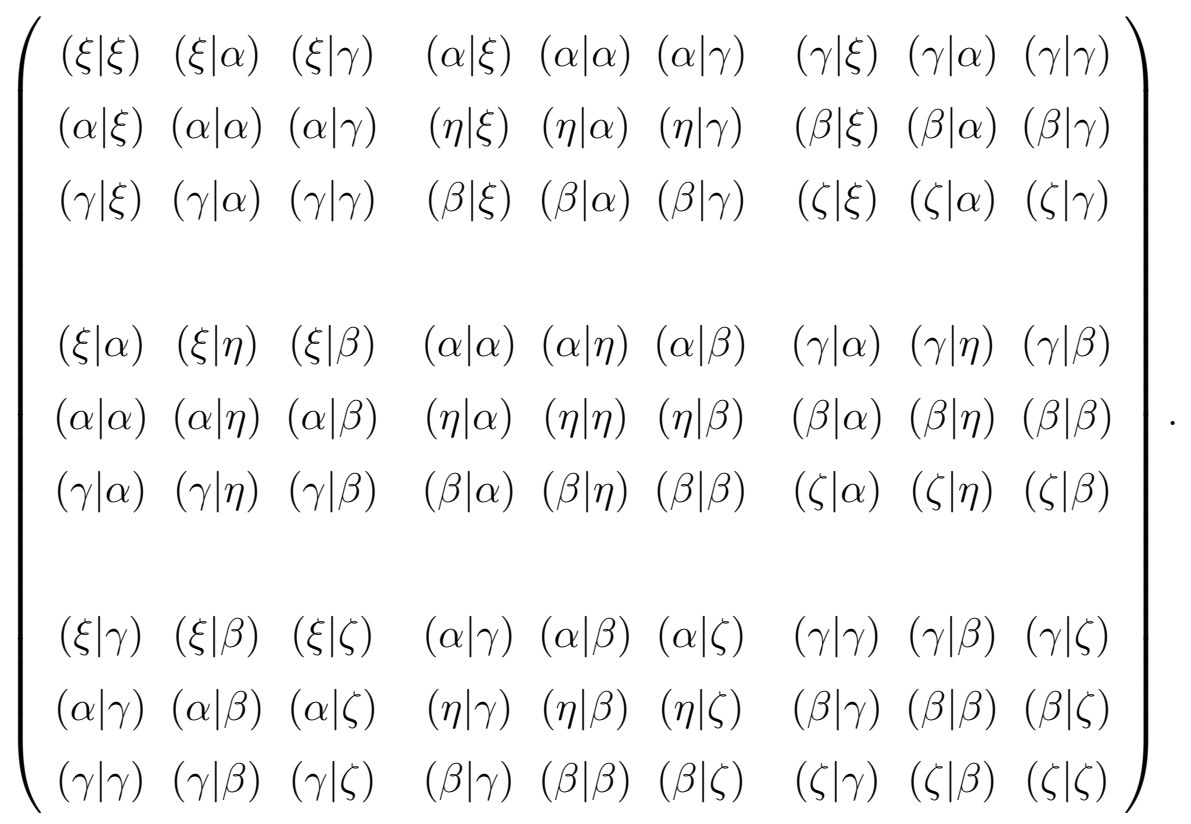


Since $(1,0,0 ; 0,1,0 ; 0,0,1)^{\mathrm{t}}$ is in the $\operatorname{Ker} A \subseteq \operatorname{Ker} X$, we have

$$
\|\xi\|^{2}=e^{i \theta}\|\alpha\|^{2}+e^{-i \theta}\|\gamma\|^{2}, \quad\|\eta\|^{2}=e^{i \theta}\|\beta\|^{2}+e^{-i \theta}\|\alpha\|^{2}, \quad\|\zeta\|^{2}=e^{i \theta}\|\gamma\|^{2}+e^{-i \theta}\|\beta\|^{2},
$$

and so we have $\|\alpha\|=\|\beta\|=\|\gamma\|$. If $\|\alpha\|=\|\beta\|=\|\gamma\|=0$, then $A=0$. So, we may assume that

$$
\|\alpha\|=\|\beta\|=\|\gamma\|=1
$$

Then we have

$$
\|\xi\|^{2}=\|\eta\|^{2}=\|\zeta\|^{2}=e^{i \theta}+e^{-i \theta} .
$$

Considering $(2,4),(6,8)$ and $(7,3)$ principal submatrices, we also have

$$
|(\xi \mid \eta)| \leq 1, \quad|(\eta \mid \zeta)| \leq 1, \quad|(\zeta \mid \xi)| \leq 1
$$

If we take vectors so that $\operatorname{span}\{\xi, \eta, \zeta\} \perp \operatorname{span}\{\alpha, \beta, \gamma\}$ with mutually orthonormal vectors $\alpha, \beta, \gamma$ then we have

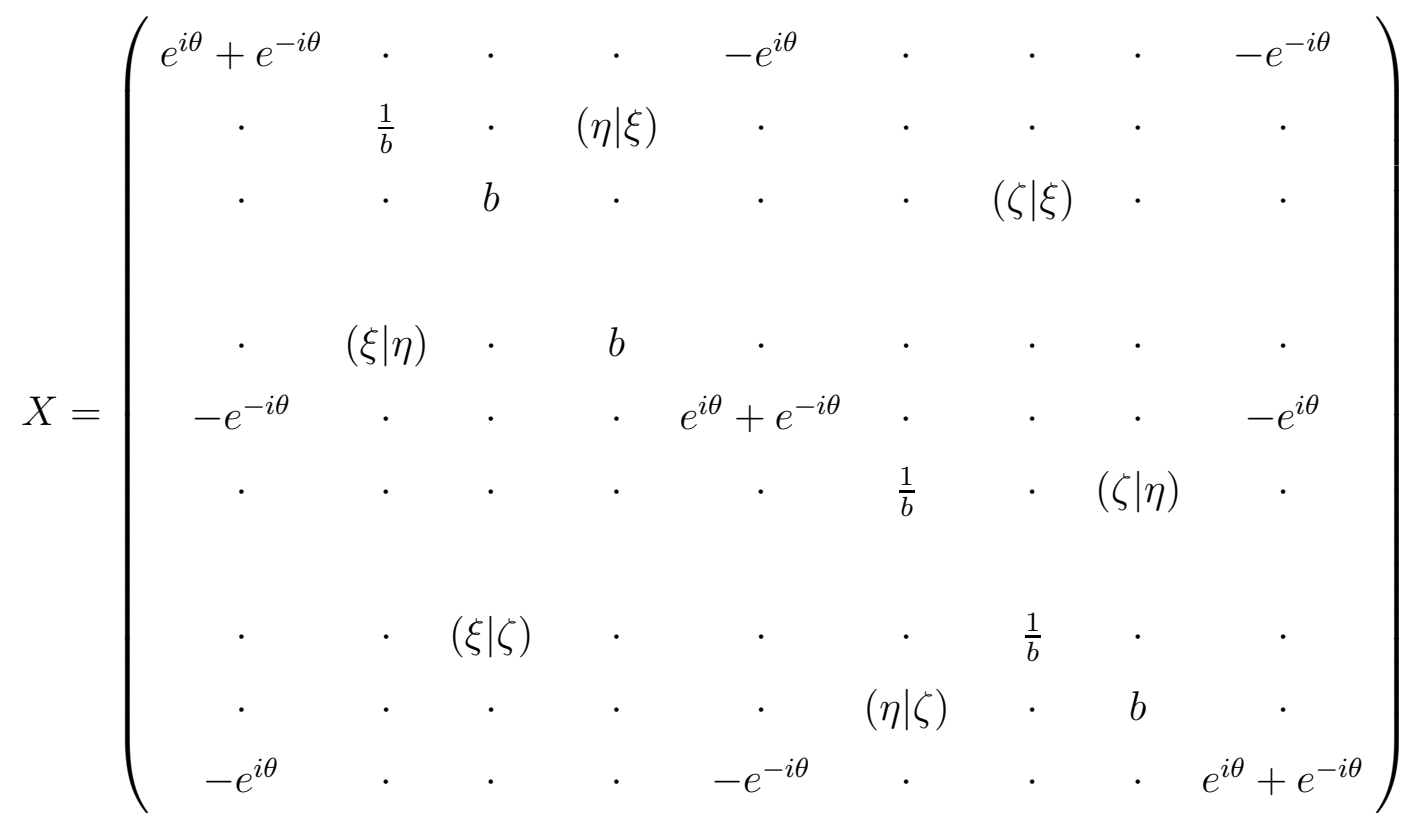


and

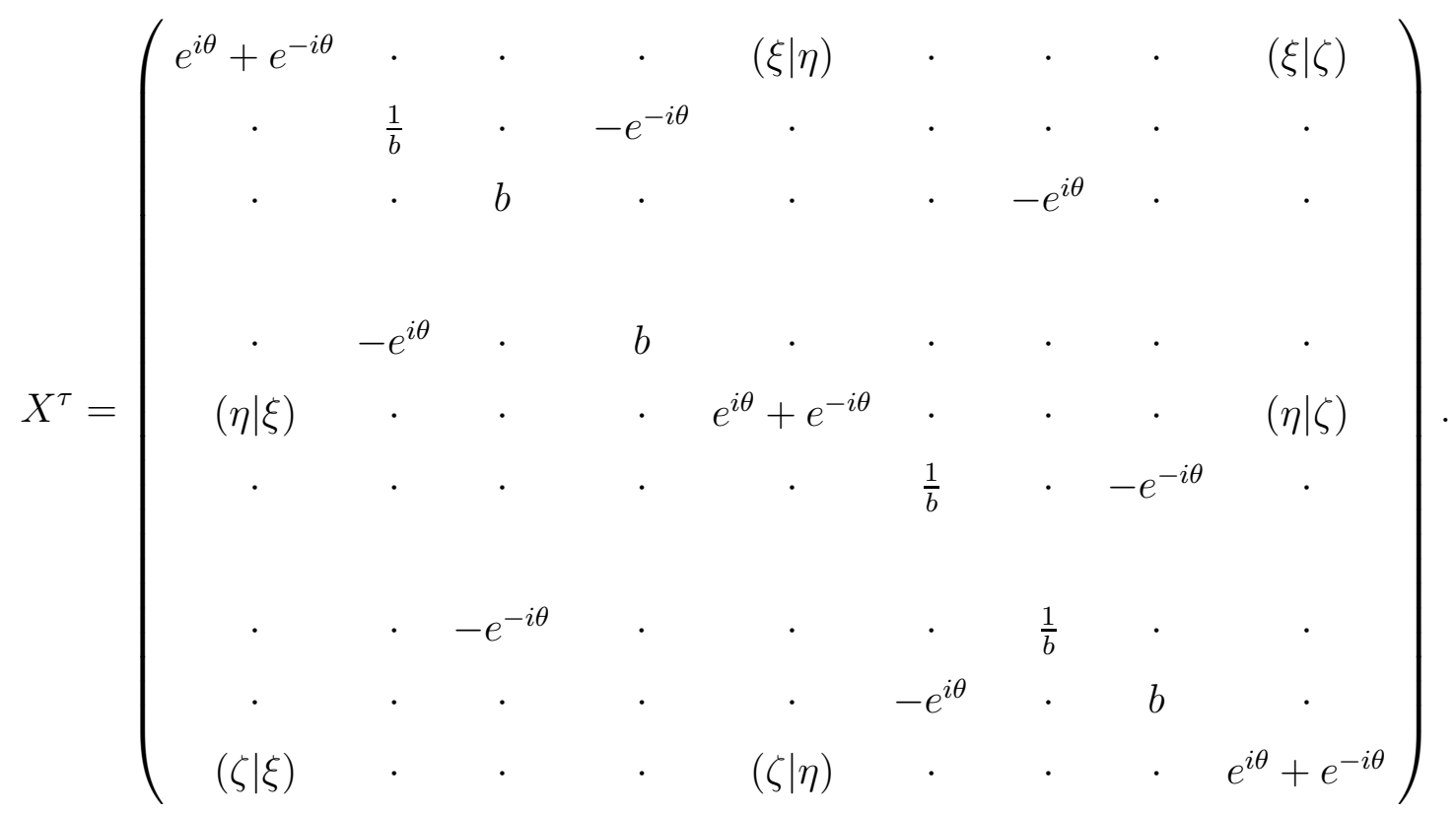

It is clear that $X$ is of PPT under the conditions (7) and (8). We note that the rank of $X$ is equal to

$$
2+\operatorname{rank}\left(\begin{array}{cc}
\frac{1}{b} & (\xi \mid \eta) \\
(\eta \mid \xi) & b
\end{array}\right)+\operatorname{rank}\left(\begin{array}{cc}
\frac{1}{b} & (\eta \mid \zeta) \\
(\zeta \mid \eta) & b
\end{array}\right)+\operatorname{rank}\left(\begin{array}{cc}
b & (\zeta \mid \xi) \\
(\xi \mid \zeta) & \frac{1}{b}
\end{array}\right)
$$

and the rank of $X^{\tau}$ is equal to

$$
3+\operatorname{rank}\left(\begin{array}{ccc}
e^{i \theta}+e^{-i \theta} & (\xi \mid \eta) & (\xi \mid \zeta) \\
(\eta \mid \xi) & e^{i \theta}+e^{-i \theta} & (\eta \mid \zeta) \\
(\zeta \mid \xi) & (\zeta \mid \eta) & e^{i \theta}+e^{-i \theta}
\end{array}\right)=3+\operatorname{dim} \operatorname{span}\{\xi, \eta, \zeta\}
$$

In the three dimensional space $\mathbb{C}^{3}$, it is possible to take linearly independent vectors $\xi, \eta, \zeta$ satisfying (7) and (8) so that some of

$$
(\xi \mid \eta), \quad(\eta \mid \zeta), \quad(\zeta \mid \xi)
$$

are of absolute values one and the remainders are zero. Therefore, we get examples of edge states of types $(8,6),(7,6),(6,6)$ and $(5,6)$.

To get edge states of type $(p, 5)$ for $p=5,6,7,8$, it is convenient to consider the matrix

$$
P[\rho, \sigma, \tau]:=\left(\begin{array}{ccc}
e^{i \theta}+e^{-i \theta} & \rho & \bar{\tau} \\
\bar{\rho} & e^{i \theta}+e^{-i \theta} & \sigma \\
\tau & \bar{\sigma} & e^{i \theta}+e^{-i \theta}
\end{array}\right)
$$


with the conditions

$$
|\rho| \leq 1, \quad|\sigma| \leq 1, \quad|\tau| \leq 1, \quad \operatorname{det} P[\rho, \sigma, \tau]=0
$$

Then $P[\rho, \sigma, \tau]$ is a positive semi-definite matrix of rank two. By spectral decomposition, we may get two vectors $E_{1}=\left(\xi_{1}, \eta_{1}, \zeta_{1}\right)$ and $E_{2}=\left(\xi_{2}, \eta_{2}, \zeta_{2}\right)$ so that $P[\rho, \sigma, \tau]$ is the sum of rank one projectors onto $E_{1}$ and $E_{2}$, respectively. Then we see that

$$
P[\rho, \sigma, \tau]=\left(\begin{array}{ccc}
\left|\xi_{1}\right|^{2} & \xi_{1} \bar{\eta}_{1} & \xi_{1} \bar{\zeta}_{1} \\
\eta_{1} \bar{\xi}_{1} & \left|\eta_{1}\right|^{2} & \eta_{1} \bar{\zeta}_{1} \\
\zeta_{1} \bar{\xi}_{1} & \zeta_{1} \bar{\eta}_{1} & \left|\zeta_{1}\right|^{2}
\end{array}\right)+\left(\begin{array}{ccc}
\left|\xi_{2}\right|^{2} & \xi_{2} \bar{\eta}_{2} & \xi_{2} \bar{\zeta}_{2} \\
\eta_{2} \bar{\xi}_{2} & \left|\eta_{2}\right|^{2} & \eta_{2} \bar{\zeta}_{2} \\
\zeta_{2} \bar{\xi}_{2} & \zeta_{2} \bar{\eta}_{2} & \left|\zeta_{2}\right|^{2}
\end{array}\right)=\left(\begin{array}{ccc}
(\xi \mid \xi) & (\xi \mid \eta) & (\xi \mid \zeta) \\
(\eta \mid \xi) & (\eta \mid \eta) & (\eta \mid \zeta) \\
(\zeta \mid \xi) & (\zeta \mid \eta) & (\zeta \mid \zeta)
\end{array}\right) .
$$

If we take $\rho, \sigma, \tau$ with (9) so that some of them are of absolute values one and the remainders of them have the absolute values less than one, then we get PPT entangled states of types $(8,5),(7,5),(6,5)$ and $(5,5)$, as we will now show.

For a given fixed $\theta$ with (3), we can take a real number $r$ with $-1<r<1$ so that

$$
P[r, r, r], \quad P[r,-r, 1], \quad P[1,1, r]
$$

is of rank two, respectively, to get edge states of types $(8,5),(7,5)$ and $(6,5)$. For example, we see that

$$
P[-\cos \theta,-\cos \theta,-\cos \theta]
$$

is of rank two, and so we get the following natural examples of $3 \otimes 3$ edge states of type $(8,5)$ :

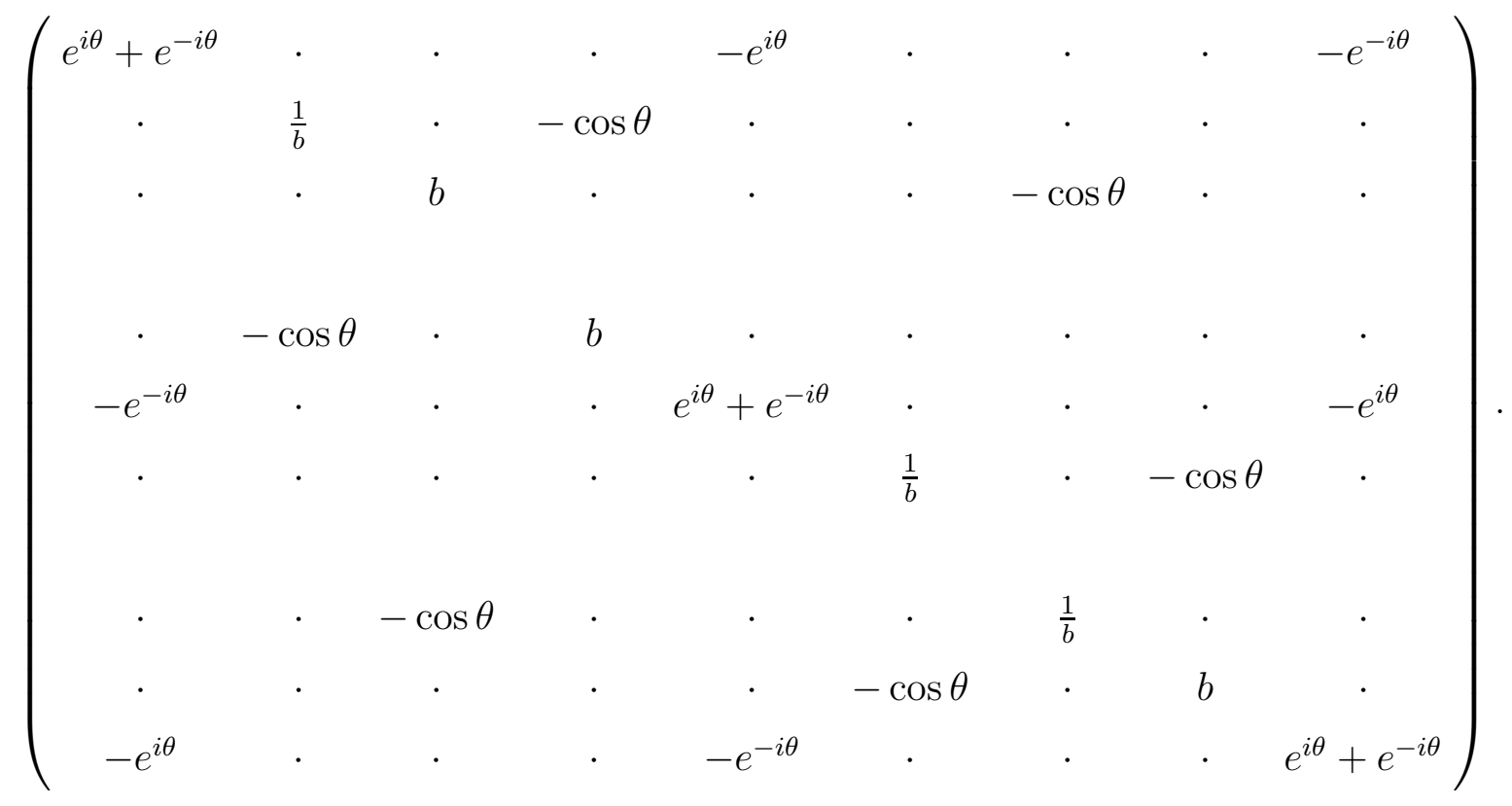


To get examples of edge states of types $(7,5)$ and $(6,5)$, we put $\omega=e^{i \theta}+e^{-i \theta}$ temporarily. Note that $1<\omega<2$. We also note that

$$
\operatorname{det} P[r,-r, 1]=(1+\omega)\left(\omega^{2}-\omega-2 r^{2}\right), \quad \operatorname{det} P[1,1, r]=(\omega-r)\left(r \omega+\omega^{2}-2\right) \text {. }
$$

and zeros

$$
r=\sqrt{\frac{\omega^{2}-\omega}{2}}=\sqrt{2 \cos ^{2} \theta-\cos \theta}, \quad r=\frac{2-\omega^{2}}{\omega}=-\frac{\cos 2 \theta}{\cos \theta}
$$

of them are in the interval $(-1,1)$, respectively. In this way, we get edge states of types $(7,5)$ and $(6,5)$. If we consider the rank two matrix $P\left[-e^{i \theta},-e^{i \theta},-e^{i \theta}\right]$, which is nothing but $P[\theta]$ at the beginning of the construction, then we have the following parameterized example of edge states of type $(5,5)$ :

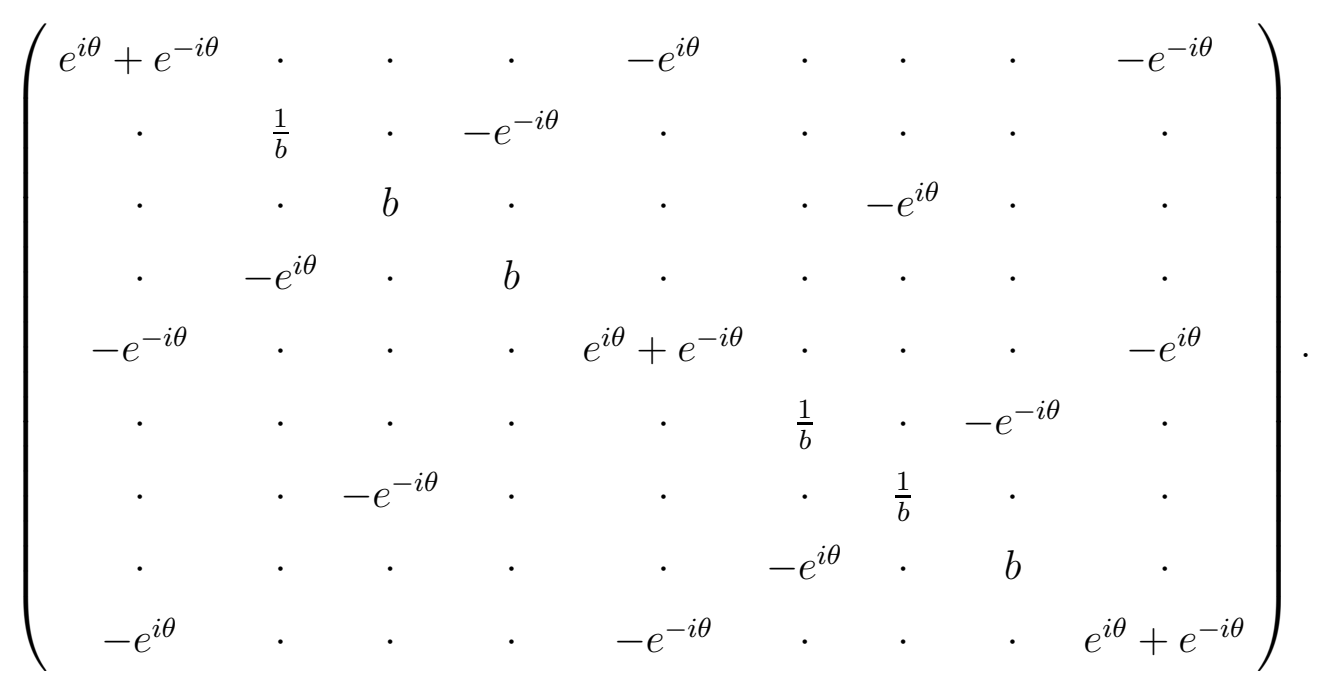

In conclusion, we have constructed $3 \otimes 3$ PPT entangled edge states of type $(8,6)$ whose existence has been a long-standing question since the claim in Ref. 26 without proof. In this vein, it would be also an interesting question whether there exists a $2 \otimes 4$ edge states of type $(6,6)$ or not, as was explained in Ref. 20. We have shown that there exist edge states of all possible types in the face generated by each PPT state we constructed, except for edge states of $(4,4)$ types. These include parameterized examples of edge states of types $(5,5)$ and $(6,6)$, for which there have been known very few discrete examples $\underline{10} \underline{11}$. We also have natural parameterized examples of edge states of type $(8,5)$. Compare with Ref. 13. We note that the study of bi-qutrit edge states with minimal ranks was initiated by Ref. 3 , and have been recently studied in Ref. 5, 15, 28, and 29 very extensively. It is the authors' hope that this is the starting point for the further study of bi-qutrit edge states with maximal ranks. 
SHK was partially supported by NRFK 2011-0001250. HO was partially supported by the JSPS grant for Scientific Research No.20540220. The first author is grateful to Kil-Chan Ha for helpful discussion.

\section{REFERENCES}

${ }^{1}$ E. Alfsen and F. Shultz, Unique decompositions, faces, and automorphisms of separable states, J. Math. Phys. 51(2010), 052201.

${ }^{2} \mathrm{~T}$. Ando, Cones and norms in the tensor product of matrix spaces, Linear Algebra Appl. 379 (2004), 3-41.

${ }^{3}$ C. H. Bennett, D. P. DiVincenzo, T. Mor, P. W. Shor, J. A. Smolin, and B. M. Terhal, Unextendible product bases and bound entanglement, Phys. Rev. Lett. 82 (1999), 53855388.

${ }^{4}$ E.-S. Byeon and S.-H. Kye, Facial structures for positive linear maps in the two dimensional matrix algebra, Positivity 6 (2002), 369-380.

${ }^{5}$ L. Chen and D. Ž. Djoković, Description of rank four PPT entangled states of two qutrits, J. Math. Phys. 52 (2011), 122203.

${ }^{6}$ S.-J. Cho, S.-H. Kye, and S. G. Lee, Generalized Choi maps in 3-dimensional matrix algebras, Linear Alg. Appl. 171 (1992), 213-224.

${ }^{7}$ H.-S. Choi and S.-H. Kye, Facial structures for separable states, J. Korean Math. Soc., 49 (2012), to appear, http://www.math.snu.ac.kr/ kye/paper/separable.pdf.

${ }^{8}$ M.-D. Choi, Completely positive linear maps on complex matrices, Linear Alg. Appl. 10 (1975), 285-290.

${ }^{9}$ M.-D. Choi, Positive linear maps, Operator Algebras and Applications (Kingston, 1980), pp. 583-590, Proc. Sympos. Pure Math. Vol 38. Part 2, Amer. Math. Soc., 1982.

${ }^{10}$ L. Clarisse, Construction of bound entangled edge states with special ranks, Phys. Lett. A 359 (2006), 603-607.

${ }^{11}$ K.-C. Ha, Comment on : "Construction of bound entangled edge states with special ranks" [Phys. Lett. A 359 (2006) 603], Phys. Lett. A 361 (2007), 515-519.

${ }^{12}$ K.-C. Ha and S.-H. Kye, Construction of entangled states with positive partial transposes based on indecomposable positive linear maps, Phys. Lett. A 325 (2004), 315-323.

${ }^{13}$ K.-C. Ha and S.-H. Kye, Construction of $3 \otimes 3$ entangled edge states with positive partial 
transposes, J. Phys. A 38 (2005), 9039-9050.

${ }^{14}$ K.-C. Ha, S.-H. Kye, and Y. S. Park, Entanglements with positive partial transposes arising from indecomposable positive linear maps, Phys. Lett. A 313 (2003), 163-174.

${ }^{15}$ L. O. Hansen, A. Hauge, J. Myrheim, and P. Ø. Sollid, Low rank positive partial transpose states and their relation to product vectors, preprint, arXiv:1104.1519.

${ }^{16} \mathrm{M}$. Horodecki, P. Horodecki and R. Horodecki, Mixed-state entanglement and distillation: is there a "bound" entanglement in nature?, Phys. Rev. Lett. 80 (1998), 5239-5242.

${ }^{17}$ M. Horodecki, P. W. Shor and M. B. Ruskai, General entanglement braking channels, Rev. Math. Phys. 15 (2003), 629-641.

${ }^{18} \mathrm{P}$. Horodecki, Separability criterion and inseparable mixed states with positive partial transposition, Phys. Lett. A 232 (1997), 333-339.

${ }^{19}$ P. Horodecki, M. Lewenstein, G. Vidal, and I. Cirac, Operational criterion and constructive checks for the separability of low rank density matrices, Phys. Rev. A 62 (2000), 032310.

${ }^{20}$ Y.-H. Kiem, S.-H. Kye, and J. Lee, Existence of product vectors and their partial conjugates in a pair of spaces, J. Math. Phys, 52 (2011), 122201.

${ }^{21}$ A. S. Kholevo, M. E. Shirokov and R. F. Werner, On the notion of entanglement in Hilbert spaces, Russian Math. Surveys 60 (2005), 359-360.

${ }^{22}$ J. M. Leinaas, J. Myrheim, and P. Ø. Sollid, Numerical studies of entangled PPT states in composite quantum systems, Phys. Rev. A 81 (2010), 062329.

${ }^{23}$ M. Lewenstein, B. Kraus, P. Horodecki, and J. I. Cirac, Characterization of separable states and entanglement witnesses, Phys. Rev. A 63 (2001), 044304.

${ }^{24}$ B. Kraus, J. I. Cirac, S. Karnas, and M. Lewenstein, Separability in $2 \mathrm{xN}$ composite quantum systems, Phys. Rev. A 61 (2000), 062302.

${ }^{25}$ A. Peres, Separability criterion for density matrices, Phys. Rev. Lett. 77 (1996), 14131415.

${ }^{26}$ A. Sanpera, D. Bruß, and M. Lewenstein, Schmidt-number witnesses and bound entanglement, Phys. Rev. A 63 (2001), 050301.

${ }^{27}$ A. I. Singh, Quantum Dynamical Semigroups involving Separable and Entangled States, preprint, arXiv:1201.0250.

${ }^{28} \mathrm{E}$. Skowronek, Three-by-three bound entanglement with general unextendible product bases, J. Math. Phys. 52 (2011), 122202.

${ }^{29}$ P. Ø. Sollid, J. M. Leinaas, and J. Myrheim, Unextendible product bases and extremal 
density matrices with positive partial transpose, Phys. Rev A 84 (2011), 042325.

${ }^{30}$ E. Størmer, Positive linear maps of operator algebras, Acta Math. 110 (1963), 233-278.

${ }^{31}$ E. Størmer, Decomposable positive maps on $C^{*}$-algebras, Proc. Amer. Math. Soc. 86 (1982), 402-404.

${ }^{32}$ N. R. Wallach, An Unentangled Gleason's Theorem, Contemp. Math. 305 (2002), 291-298.

${ }^{33}$ S. L. Woronowicz, Positive maps of low dimensional matrix algebras, Rep. Math. Phys. 10 (1976), 165-183. 